\title{
Etude de critères de sélection chez le maïs fourrage : héritabilités, corrélations génétiques et réponse attendue à la sélection
}

André GALLAIS (*), Patrick VINCOURT \& Jean-Claude BERTHOLLEAU

I.N.R.A., Station d'amélioration des Plantes fourragères, F 86600 Lusignan

(*) Nouvelle adresse: Institut National Agronomique, chaire de génétique, 16 rue Claude Bernard, F 75005, Paris

La variabilité génétique et environnementale pour différents critères de sélection du maïs fourrage a été étudiée au niveau des descendances top cross (avec un testeur hybride simple) de 6 synthétiques ( 4 cornées, 2 dentées), le testeur étant différent pour chaque synthétique. Les caractères étudiés étaient les suivants : vigueur au départ, précocité de floraison, hauteur des plantes, hauteur de l'épi, longueur et largeur de la feuille de l'épi, rendement en matière sèche, teneur en matière sèche de la plante entière, teneurs en ADF, protéines, cellulose et glucides solubles. Les caractères de développement et de morphologie apparaissent en général très héritables; le rendement et les teneurs en ADF et protéines sont les moins héritables. Certaines corrélations génétiques sont apparues assez stables et assez élevées : corrélation positive entre rendement et hauteur de l'épi ou de la plante, corrélation négative entre rendement et facteurs de la qualité (teneur en protéines, teneur en $\mathrm{ADF}$ ). Les conséquences au niveau de la réponse à la sélection sont envisagées. En vue d'une sélection pour le rendement à précocité donnée, il apparaît particulièrement efficace d'associer aux mesures de rendement une notation de la vigueur à un stade précoce et les mesures de hauteur de la plante ou de l'épi avec une pondération biométrique des caractères, en fonction des héritabilités et des corrélations génétiques et environnementales entre les caractères considérés.

Mots clés additionnels : Zea mays, rendement en matière secche, valeur alimentaire, sélection indirecte, index de sélection, sélection multicaractères.

Study of selection criteria in forage maize : heritabilities, genetic correlations and expected response to selection.

Genetic and environmental variability was studied for different selection criteria in forage maize at the level of top-cross progenies (with a single cross tester) for 6 synthetics ( 4 flint, 2 dent), the tester being different for each synthetic. The following characters were studied: early vigour, flowering earliness, plant and ear heights, length and width of ear leaf, dry matter yield, dry matter content of the whole plant and aciddetergent fiber (ADF), protein, cellulose, and soluble sugar contents. Characters related to earliness and to morphology had high heritability ; dry matter yield, protein and ADF contents had the lowest heritability. High and stable genetic correlations were observed between yield of the whole plant and either ear or plant height (positive correlation), and between yield and factors influencing feeding value (negative correlation with protein content, positive correlation with ADF content). Consequences for the response to selection on different criteria are discussed. For the improvement of yield at a given earliness, it appears very efficient to combine the measurements of yield and plant (or ear) height with a score for young plant vigour, with a biometrical weighting of the characters, taking into account heritabilities and genetic and phenotypic correlations between the characters.

Additional key words : Zea mays, dry matter yield, feeding value, indirect selection, selection index, multitrait selection.

\section{INTRODUCTION}

De l'ensemble des travaux réalisés sur les critères de sélection du maïs fourrage, et plus particulièrement de ceux réalisés à la Station d'amélioration des Plantes fourragères de Lusignan, certaines conclusions assez claires semblent pouvoir être tirées en ce qui concerne les critères de rendement et de qualité (Gallais et al., 1976, 1981). Pour avoir le rendement en matière sèche maximum, même à précocité donnée, il faut un développement de toutes les parties de la plante et, en particulier, de la tige. Pour améliorer la valeur alimentaire, il apparaît qu'il faut dimi- 
nuer les teneurs en fibres et en cellulose, augmenter les teneurs en protéines et en glucides, le meilleur critère de la digestibilité et de la quantité ingérée apparaissant être la digestibilité in vitro. Toutefois, la plupart de ces conclusions reposent sur des études de corrélations phénotypiques entre caractères, avec du matériel assez varié (hybrides expérimentaux). C'est, en général, la première étape d'une étude de critères de sélection.

Dans une deuxième étape, il est indispensable de vérifier que, dans le matériel en cours de sélection, ces corrélations existent toujours et ont le sens de corrélations génétiques et que les caractères retenus sont sulfisamment héritables. Il faut de plus vérifier que les objectifs sont réalistes. Par exemple, sera-t-il possible de cumuler progrès en rendement et progrès en qualité ?

Cette étude a donc pour but de préciser la valeur de différents critères de sélection et les possibilités de sélection pour le rendement et pour la qualité au niveau de populations synthétiques qui ont été constituées à Lusignan pour la mise en place d'une sélection récurrente. Elle repose essentiellement sur l'analyse de la variabilité phénotypique et génotypique d'où sont tirées les estimations des héritabilités des différents caractères et les corrélations génétiques et environnementales entre caractères. Ces paramètres permettront, en particulier, de préciser l'intérêt de la prise en considération de plusieurs caractères pour la sélection sur un seul caractère.

\section{MATÉRIEL ET MÉTHODES}

\section{A. Préparation du matériel}

Différentes synthétiques (tabl. 1) ont été développées à partir de lignées «classiques " déjà sélectionnées pour le grain, d'origines variées mais en général plus précoces que la lignée W 182 E, l'objectif étant la création d'un matériel mais fourrage assez précoce. Pour préserver l'aptitude à la combinaison connue entre les groupes corné curopéen et denté nord-américain, ces 2 groupes ont été respectés. A l'intérieur de chaque groupe, des synthétiques par « objectif " ont été formées, sur la base des résultats du croisement de chaque lignée avec 2 à 4 testeurs : les lignées manifestant une bonne aptitude à la combinaison respectivement pour le rendement, pour des critères de valeur alimentaire, pour la teneur en protéines sont entrées dans les synthétiques rendement, qualité et teneur en protéines. L'intercroise- ment a été réalisé de façon manuelle, selon un schéma de type circulaire (fig. 1) jusqu'au niveau hybride double.

A chaque synthétique, un testeur hybride simple, corné pour les synthétiques dentées et denté pour les synthétiques cornées, a été associé selon certains critères de valeur en combinaison (bonne valeur en combinaison avec la synthétique considérée pour différents caractères de valeur fourragère en privilégiant l'objectif de la synthétique). Ce testeur est utilisé pour une sélection récurrente à testeur constant.

Pour l'appréciation de la valeur génétique du matériel, et donc de la variabilité à l'intérieur de chaque synthétique, 150 à 300 plantes ont été autofécondées et croisées en même temps avec le testeur. Ces plantes étaient choisies parmi les plus précoces et les plus vigoureuses, mais de telle sorte qu'il y ait autant de plantes prises à l'intérieur de chaque

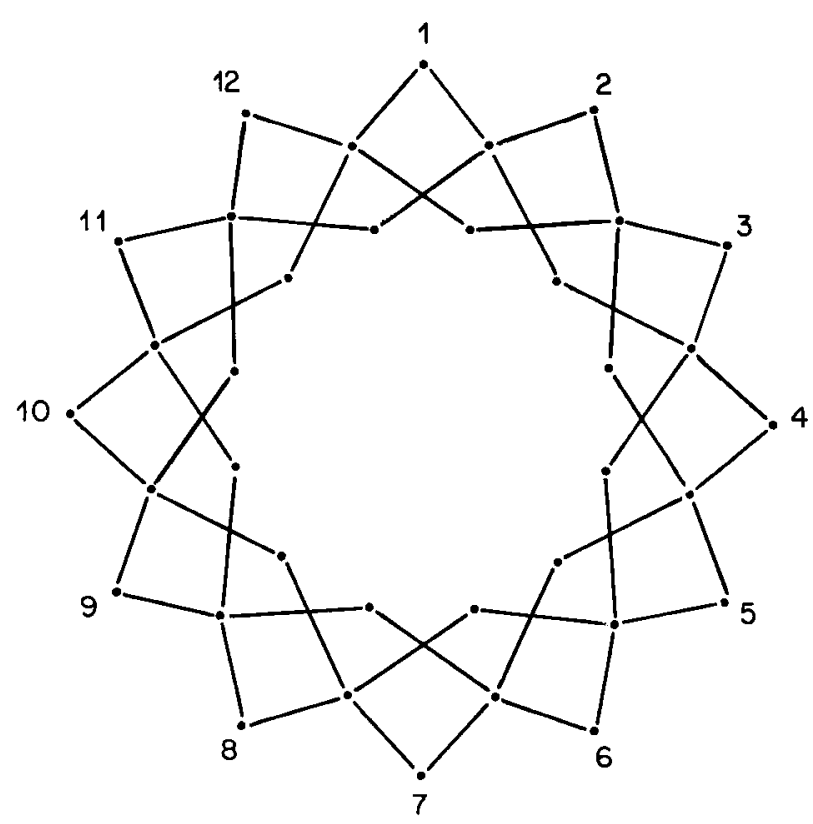

Figure 1

Schéma de l'intercroisement des lignées pour la production des synthétiques (croisement en chaîne des lignées $1 \times 2 \times 3 \ldots$ pour produire les hybrides simples et croisement des hybrides simples successifs non apparentés pour produire les hybrides doubles: $(1 \times 2) \times(3 \times 4) \ldots$

System of intercrossing the lines to produce synthetics (chain cross $1 \times 2 \times 3$ to produce single crosses and crossing of unrelated successive single crosses to produce double crosses: $(1 \times 2) \times(3 \times 4) \ldots$

TABLEAU 1

Description sommaire du matériel utilisé.

Description of the material.

\begin{tabular}{|c|c|c|c|c|}
\hline $\begin{array}{l}\text { Synthétique } \\
\text { Synthetic }\end{array}$ & $\begin{array}{c}\text { Texture } \\
\text { du grain }\left(^{*}\right) \\
\text { Type of grain }\end{array}$ & $\begin{array}{l}\text { Nombre de } \\
\text { lignćcs } \\
\text { Number } \\
\text { of lines }\end{array}$ & $\begin{array}{l}\text { Nombre de } \\
\text { descendances } \\
\text { Number of } \\
\text { progenies }\end{array}$ & $\begin{array}{c}\text { Année } \\
\text { d'étude } \\
\text { Year } \\
\text { of test }\end{array}$ \\
\hline Syn 20 & $\mathrm{D}$ & 29 & 228 & 1981 \\
\hline Syn $4 \times 14$ & D & 12 & 215 & 1980 \\
\hline Syn 19 & C & 25 & 278 & 1981 \\
\hline Syn $3 \times 13$ & $\mathrm{C}$ & 13 & 130 & 1980 \\
\hline Syn 21 & $\mathrm{C}$ & 14 & 158 & 1980 \\
\hline Syn $5 \times 9$ & $\mathrm{C}$ & 15 & 399 & 1981 \\
\hline
\end{tabular}

(*) D = Denté =: Dent.

$\mathrm{C}=$ Comé $=$ Flint . 
hybride double constituant la synthétique, afin de limiter la dérive. Ce qui sera étudié, ce n'est donc pas la variabilité totale de la synthétique, mais la variabilité de la «sous population » ainsi échantillonnée.

\section{B. Dispositif expérimental}

Les descendances en croisement avec le testeur ont été installées dans un dispositif lattice à 2 répétitions dans 2 lieux, dont toujours la Station de Lusignan. Nous ne présentons ici que les résultats obtenus dans le lieu «Lusignan $»$.

Le dispositif expérimental est formé par un ensemble de lattices $8 \times 8$ ou $8 \times 9$ : les parcelles sont formées d'une ligne de $5 \mathrm{~m}$, espacées de $80 \mathrm{~cm}$, la densité étant de 85000 plantes/ha.

\section{Caractères observés}

Dans ce qui suit, la notation des caractères indiquée entre parenthèses est celle qui est utilisée dans les tableaux.

1. La vigueur au départ (VIG) : notation visuelle de la vigucur réalisée de 1 à 5 , au stade 7-9 feuilles visibles.

2. La floraison femelle (FLOR) : appréciée par la date à laquelle 50 p. 100 des soies sont sorties (non observée sur les synthétiques 19 et 20 ).

3. Caractères morphologiques observés après la floraison:

- Hauteur totale de la plante (HP) et hauteur de l'épi (HE) ; la hautcur totale est mesurée du sol à la base de la $1^{\text {re }}$ ramification de la panicule et la hauteur de l'épi est mesurée du sol au noud de l'épi supéricur.

- Dimensions de la feuille de l'épi : longueur (LONG) et largeur (LARG) mesurée dans la zone la plus large (ticrs inféricur).

4. Caractères observés à la récolte:

La récolte a cu lieu essai par essai ou par groupe d'essais, pour des tencurs en matières sèches variant de 27 à 40 p. 100 selon les essais. A la récolte, la matière verte totale par parcelle est pesée et la teneur en matière sèche (p. $100 \mathrm{MS}$ ) est appréciéc par prélèvement de $1 \mathrm{~kg}$ de matière verte par parcelle séchéc à l'ćtuve à $85^{\circ} \mathrm{C}$ pendant $24 \mathrm{~h}$. De ces 2 observations, il résulte une estimation du rendement en matière sèche (RDT).

5. Caractères de composition chimique :

Trois caractères de composition chimique ont été étudiés à l'aide de l'infra-analyseur NEOTEC (F.Q.A. 51): la teneur en ADF (Acid Detergent Fiber = lignine + cellulose), la tencur en protéines (PROT) et la teneur en glucides solubles (GLU). Ces caractères n'ont pas été observés sur les synthétiques 19 et 20.

Compte tenu de la technologic employée pour évaluer ces composantes qualitatives, les corrélations phénotypiques ct génotypiques estimées surestiment vraiscmblablement, en valcur absolue, les valeurs réclles. En effet le faible nombre de filtres utilisés et efficaces conduit à des corrélations fortes entre estimateurs des caractéristiques d'un même échantillon.

\section{Interprétation}

La structure en lattice n'a pas été prise en considération pour l'estimation des composantes de la variance. L'analyse de variance multivariable conduit à l'estimation des matrices de variances-covariances entre familles et résiducle (voir annexe). Ces estimations permettent de calculer les héritabilités entre familles (rapport de la variance génétique entre familles à la variance phénotypique totale entre parcelies) et les corrélations génétiques et environnementales, ou plus exactement résiduelles, entre caractères. Cette analyse a ćté prolongée par le calcul de la valeur génotypique $\left(G_{i, P}\right)$ de chaque famille pour chaque caractère en tenant compte des relations entre caractères. Celle-ci est définie par la régression de la valcur génotypique $G_{i}$ d'un caractère $q$ sur les valeurs phénotypiques $P_{i}$ de tous les caractères mesurés, utilisćs comme prédicteurs (ROUVIER, 1969; Gallais, 1973):

$$
{ }_{q} G_{i p}={ }_{q} \Sigma_{g p} \Sigma_{p p}^{-1}\left(P_{i}\right)
$$

${ }_{\mathrm{q}} \Sigma_{\mathrm{gp}}$ représentant le vecteur ligne des covariances entre la variable à prévoir et les prédicteurs $\mathrm{P}$. C'est la ligne $\mathrm{q}$ de la matrice $\Sigma_{\mathrm{qp}}$ de variances-covariances génétiques.

$\Sigma_{\text {pp }}$ est la matrice des variances-covariances phénotypiques et $(\mathrm{P})$ le vecteur colonne des variables prédictrices. Les matrices $\Sigma_{g p}$ et $\Sigma_{p p}$ sont alors remplacées par leurs estimations données par l'analyse de variance multivariable. Ces estimations ont été réalisées essai par essai puis fusionnées pour aboutir à une seule $\Sigma_{g p}$ et une seule $\Sigma_{p p}$ par synthétique.

La matrice $\Sigma_{\mathrm{gg}}^{\prime}=\Sigma_{\mathrm{gp}} \Sigma_{\mathrm{pp}}^{-1} \Sigma_{\mathrm{pg}}$ est la matrice de covariance des prédicteurs des valeurs génétiques; elle peut être comparée à la matrice $\Sigma_{\mathrm{gg}}$ de covariance des vraies valeurs génétiques, en particulier pour évaluer les distorsions susceptibles de se produire au niveau des corrélations. Le rapport des éléments diagonaux de la matrice $\Sigma_{\mathrm{gg}}^{\prime}$ à ceux de la matrice $\Sigma_{\mathrm{gg}}$ mesure le degré de précision $r_{(\mathrm{G}, \overline{\mathrm{G}})}^{2}$ de l'appréciation des valeurs génétiques par les prédicteurs phénotypiques choisis. C'est une héritabilité au sens multivariable $\left(h_{M}^{2}\right)$, qui est à comparer à l'héritabilité au sens univariable $h_{U}^{2}$ pour mesurer l'efficacité de la prise en compte de certains caractères associés.

On distinguera dans la suite le critère $h_{U}^{2}$, qui dépend explicitement du plan d'expérience $\sigma_{\mathrm{G}}^{2} /\left(\sigma_{\mathrm{G}}^{2}+\frac{\sigma_{2}^{2}}{\mathrm{~L}}\right)$, où $\mathrm{L}$ est le nombre de blocs, du critère $\mathrm{h}^{2}$ qui mesure, pour un caractère donné, la corrélation entre 2 répétitions de la même famille $\left[h^{2}=\sigma_{\mathrm{G}}^{2} /\left(\sigma_{\mathrm{G}}^{2}+\sigma_{\mathrm{e}}^{2}\right)\right]$.

Le progrès génétique $(\Delta \mathrm{G})$ attendu par sélection sur un seul caractère (q) en tenant compte des liaisons entre caractères est déduit des estimations des variances-covariances génotypiques et phénotypiques:

$$
\begin{aligned}
\Delta \mathrm{G}_{\mathrm{q}} & =\mathrm{i} \sqrt{\operatorname{var} \overline{\bar{G}}} \\
& =\mathrm{i}\left({ }_{\mathrm{q}} \Sigma_{\mathrm{gp}} \Sigma_{\mathrm{pp}}^{-1} \Sigma_{\mathrm{q}} \Sigma_{\mathrm{gp}}\right)^{1 / 2}
\end{aligned}
$$

i étant l'intensité de sélection en unités standard.

D'une façon plus générale. le vecteur des réponses des différents caractères à la sćlection sur un caractère $q$ a pour expression :

$$
(\Delta \mathrm{G} / \mathrm{q})=\left(\mathrm{i} / \sqrt{\operatorname{var} \bar{G}_{\mathrm{q}}}\right) \Sigma_{\mathrm{gp}} \Sigma_{p \mathrm{p}}^{1}{ }_{\mathrm{q}} \Sigma_{\mathrm{gp}}
$$

Les progrès génétiques ainsi calculés expriment la différence attendue entre la moyenne des familles sélectionnées et la moyenne de l'ensemble des familles. Cependant, comme la variance entre familles représente la variance d'aptitude à la combinaison avec le testcur et que celle-ci se transmet, à une partic des effets d'épistasie près, d'une génération à l'autre, ces progrès représentent aussi une prévision de la différence entre moyennes de familles de 2 génćrations successives de sélection. Il s'agit donc des progrès attendus par un cycle de sélection récurrente à testcur constant. Dans les calculs de réponse à la sćlcction, l'intensité a été prise ćgale à 2,06, c'est-à-dire pour un pourcentage d'individus sélectionnés de 5 p. 100 . 
A partir des estimations des valeurs génétiques par les différents caractères, il serait possible de passer au calcul d'un index de sélection, par simple pondération économique de ces valeurs. Le problème est de déterminer cetté pondération économique. Ce problème déjà cnvisagć par VinCOURT \& GalLaIS (1981) ne sera pas envisagé ici.

\section{RÉSULTATS ET DISCUSSION}

\section{A. L'héritabilité des différents caractères}

Du point de vue de l'héritabilité au niveau parcellaire $\left(\mathrm{h}^{2}\right)$, le tableau 2 montre qu'il est possible de faire plusieurs catégories de caractères:

- des caractères à héritabilité assez forte (de 0,30 à 0,50$)$ quelle que soit la synthétique : hauteur de l'épi, hauteur totale, longueur de feuille, précocité de floraison et p. 100 de matière sèche ;

- des caractères à héritabilité assez variable selon la synthétique : rendement en matière sèche $(0,06$ à 0,37$)$, vigueur au départ $(0,14$ à 0,50$)$, teneur en glucides $(0,17$ à $0,50)$.

Mais l'héritabilité dépend à la fois de la variance génétique et de la variance résiduelle. Il est donc important de séparer, parmi les cas où l'héritabilité est faible, ceux qui correspondent à une faible variance génétique et ceux qui correspondent à une forte variance résiduelle (tabl. 3).

La vigueur au départ montre une grande stabilité de la variation résiduelle. Les variations de $l^{\prime} h^{2}$ ne viennent donc que d'une variation de la variance génétique: ainsi les populations $4 \times 14,3 \times 13$ et 21 sont moins variables que les populations 19,20 et $5 \times 9$.

Pour les glucides, la faible $h^{2}$ associée à la population 21 vient à la fois cl'une plus faible variance génétique et d'une plus forte variance environnementale. De même, pour le rendement en matière sèche, il est remarquable que les valeurs des plus fortes $\mathrm{h}^{2}$ soient associées à une plus forte variance génétique et une plus faible variance résiduelle. Pour ce caractère, la plus faible héritabilité associée aux populations 19 et 20 vient surtout d'une plus forte variance résiduelle.

Les variations des variances résiduelles peuvent avoir une origine génétique mais aussi une origine environnementale, nécessairement plus importante dès que l'héritabilité est faible. On peut donc penser que la faible héritabilité des populations 19 et 20 pour le rendement est due à une erreur expérimentale plus grande (problème de récolte...).

Les plus fortes héritabilités correspondent à des caractères de dimension et de précocité de développement, qui se déterminent assez tôt et qui sont peut-être génétiquement plus simples. Par contre, les caractères à plus faibles héritabilités correspondent à des caractères plus complexes, résultant du fonctionnement global de la plante, plus dépendants du milieu et aussi plus difficiles à mesurer (risques d'erreurs plus grands, problèmes d'échantillonnage...). C'est le cas du rendement et des teneurs en ADF et en protéines. La teneur en glucides est un caractère très proche du développement (translocation des glucides de la tige vers le grain) et apparaît en moyenne plus héritable.

\section{B. Corrélations génétiques}

L'examen des corrélations génétiques (tabl. 4, 5, 6) ne fait pas apparaître de différences, dans les liaisons entre caractères, entre matériel denté et matériel corné. Une assez forte variation peut même être observée entre synthétiques d'un même type pour certaines corrélations (par ex. pour la relation rendement-longueur de feuilles chez le

TABLEAU 2

Héritabilités des différents caractères, au niveau parcellaire. Heritabilities of different characters at the plot level.

\begin{tabular}{|c|c|c|c|c|c|c|c|}
\hline Caractères & & $\begin{array}{c}\text { Syn } 20 \\
1981\end{array}$ & $\begin{array}{c}\text { Syn } 4 \times 14 \\
1980\end{array}$ & $\begin{array}{c}\text { Syn } 19 \\
1981\end{array}$ & $\begin{array}{c}\text { Syn } 3 \times 13 \\
1980\end{array}$ & $\begin{array}{l}\text { Syn } 21 \\
1980\end{array}$ & $\begin{array}{c}\text { Syn } 5 \times 9 \\
1981\end{array}$ \\
\hline $\begin{array}{l}\text { Vigueur Départ } \\
\text { Early vigour }\end{array}$ & VIG & 0,51 & 0,14 & 0,45 & 0,22 & 0,22 & 0,43 \\
\hline $\begin{array}{l}\text { Hauteur épi } \\
\text { Ear height }\end{array}$ & $\mathrm{HE}$ & 0,40 & 0,34 & 0,43 & 0,34 & 0,34 & 0,29 \\
\hline $\begin{array}{l}\text { Hauteur totale } \\
\text { Plant height }\end{array}$ & HT & 0,41 & 0,39 & 0,47 & 0,33 & 0,37 & 0,21 \\
\hline $\begin{array}{l}\text { Longueur feuille } \\
\text { Leaf length }\end{array}$ & LONG & 0,30 & 0,26 & 0,55 & 0,35 & 0,39 & 0,35 \\
\hline $\begin{array}{l}\text { Largeur feuille } \\
\text { Leaf width }\end{array}$ & LARG & - & 0,28 & - & 0,20 & 0,21 & - \\
\hline $\begin{array}{l}\text { Floraison } \\
\text { Flowering earliness }\end{array}$ & FLOR & - & 0,46 & - & 0,42 & 0,37 & - \\
\hline $\begin{array}{l}\text { \% MS } \\
\text { Dry matter content }\end{array}$ & $\%$ MS & 0,45 & 0,23 & 0,24 & 0,48 & 0,47 & 0,38 \\
\hline $\begin{array}{l}\text { Rendement } \\
\text { Dry matter yield }\end{array}$ & RDT & 0,06 & 0,37 & 0,13 & 0,27 & 0,15 & 0,19 \\
\hline $\begin{array}{l}\text { A.D.F. } \\
\text { A.D.F. }\end{array}$ & $\mathrm{ADF}$ & - & 0,21 & - & 0,11 & 0,06 & - \\
\hline $\begin{array}{l}\text { Protéincs } \\
\text { Protein content }\end{array}$ & PROT & - & 0,26 & - & 0,23 & 0,18 & 0,08 \\
\hline $\begin{array}{l}\text { Glucides } \\
\text { Carbohydrates }\end{array}$ & GLUC & - & 0,44 & - & 0,50 & 0,17 & - \\
\hline
\end{tabular}


TABLEAU 3

Ecarts-types génétiques $\left(\sigma_{G}\right)$ et résiduels $\left(\sigma_{W}\right)$.

Genetic $\left(\sigma_{G}\right)$ and residual $\left(\sigma_{W}\right)$ standard deviations.

\begin{tabular}{|c|c|c|c|c|c|c|c|}
\hline & & Syn 20 & Syn $4 \times 14$ & Syn 19 & Syn $3 \times 13$ & Syn 21 & Syn $5 \times 9$ \\
\hline \multirow{2}{*}{ VIG } & $\sigma_{\mathrm{G}}$ & 0,48 & 0,19 & 0,49 & 0,29 & 0,29 & 0,44 \\
\hline & $\sigma_{\mathrm{W}}$ & 0,47 & 0,46 & 0,54 & 0,55 & 0,54 & 0,51 \\
\hline \multirow{2}{*}{$\mathrm{HE}$} & $\sigma_{\mathrm{G}}$ & 5,5 & 4,7 & 6,7 & 4,6 & 4,9 & 5,0 \\
\hline & $\sigma_{W}$ & 6,7 & 6,6 & 7,6 & 6,4 & 6,8 & 7,7 \\
\hline \multirow{2}{*}{$\mathrm{HT}$} & $\sigma_{\mathrm{G}}$ & 6,3 & 7,6 & 7,8 & 6,1 & 7,1 & 6,1 \\
\hline & $\sigma_{w}$ & 7,6 & 9,3 & 8,3 & 8,6 & 9,3 & 7,7 \\
\hline \multirow{2}{*}{ LONG } & $\sigma_{\mathrm{G}}$ & 2,1 & 2,4 & 3,2 & 2,4 & 2,3 & 2,4 \\
\hline & $\sigma_{\mathrm{w}}$ & 3,1 & 4,1 & 2,9 & 3,3 & 2,9 & 3,2 \\
\hline \multirow{2}{*}{ LARG } & $\sigma_{\mathrm{G}}$ & - & 3,7 & - & 2,7 & 2,7 & - \\
\hline & $\sigma_{\mathrm{w}}$ & - & 5,9 & - & 5,4 & 5,1 & - \\
\hline \multirow{2}{*}{ FLOR } & $\sigma_{\mathrm{G}}$ & - & 1,2 & - & 1,0 & 1,0 & - \\
\hline & $\sigma_{w}$ & - & 1,3 & - & 1,1 & 1,3 & - \\
\hline \multirow{2}{*}{$\%$ MS } & $\sigma_{\mathrm{G}}$ & 1,3 & 1,1 & 1,0 & 1,4 & 1,8 & $\overline{1,3}$ \\
\hline & $\sigma_{w}$ & 1,4 & 2,0 & 1,8 & 1,5 & 1,9 & 1,7 \\
\hline \multirow{2}{*}{ RDT } & $\sigma_{\mathrm{G}}^{\prime}$ & 0,4 & 0,7 & 0,6 & 0,6 & 0,5 & 0,5 \\
\hline & $\sigma_{\mathrm{W}}$ & 1,4 & 1,0 & 1,5 & 1,0 & 1,2 & 1,1 \\
\hline \multirow{2}{*}{ A.D.F. } & $\boldsymbol{\sigma}_{\mathrm{G}}$ & - & 0,6 & - & 0,4 & 0,4 & - \\
\hline & $\sigma_{w}$ & - & 1,1 & - & 1,2 & 1,1 & - \\
\hline \multirow{2}{*}{ PROT } & $\sigma_{\mathrm{G}}$ & - & 0,3 & - & 0,2 & 0,1 & 0,2 \\
\hline & $\sigma_{w}$ & - & 0,4 & - & 0,4 & 0,3 & 0,7 \\
\hline \multirow{2}{*}{ GLUC } & $\sigma_{\mathrm{G}}$ & - & 1,9 & - & 2,0 & 1,2 & - \\
\hline & $\sigma_{w}$ & - & 2,1 & - & 2,0 & 2,7 & - \\
\hline
\end{tabular}

TABLEAU 4

Corrélations génétiques et corrélations résiduelles pour les synthétiques dentées $19^{(1)}, 3 \times 13^{(2)}, 21^{(3)}, 5 \times 9^{(4)}$. (Corrélations génétiques sous la diagonale, corrélations résiduelles au-dessus de la diagonale).

Genetic and residual correlations for dent synthetics $19^{(1)}, 3 \times 13^{(2)}, 21^{(3)}, 5 \times 9^{(4)}$. (Genetic correlations are below the diagonal).

\begin{tabular}{|c|c|c|c|c|c|c|c|c|c|c|c|c|c|c|c|c|}
\hline \multirow[b]{2}{*}{ VIG } & \multicolumn{2}{|c|}{ VIG } & \multicolumn{2}{|c|}{$\mathrm{HE}$} & \multicolumn{2}{|c|}{$\mathrm{HT}$} & \multicolumn{2}{|c|}{ LONG } & \multicolumn{2}{|c|}{ LARG } & \multicolumn{2}{|c|}{ FLOR } & \multicolumn{2}{|c|}{$\% \mathrm{MS}$} & \multicolumn{2}{|c|}{ RDT } \\
\hline & & & $\begin{array}{l}0,15 \\
0,06\end{array}$ & $\begin{array}{r}0,20 \\
-0,00\end{array}$ & $\begin{array}{r}0,23 \\
-0,02\end{array}$ & $\begin{array}{r}0,02 \\
-0,00\end{array}$ & $\begin{array}{l}0,15 \\
0,21\end{array}$ & $\begin{array}{l}0,24 \\
0,17\end{array}$ & 0,15 & $\begin{array}{l}0,10 \\
-\end{array}$ & $-0,22$ & $\begin{array}{c}-0,32 \\
-\end{array}$ & $\begin{array}{l}0,10 \\
0,25\end{array}$ & $\begin{array}{l}0,15 \\
0,21\end{array}$ & $\begin{array}{l}0,16 \\
0,11\end{array}$ & $\begin{array}{l}0,0 \\
0,17\end{array}$ \\
\hline $\mathrm{HE}$ & $\begin{array}{r}0,07^{(1)} \\
-0,03^{(2)}\end{array}$ & $\begin{array}{l}-0,15^{(3)} \\
-0,09^{(4)}\end{array}$ & & & $\begin{array}{l}0,71 \\
0,70\end{array}$ & $\begin{array}{l}0,61 \\
0,52\end{array}$ & $\begin{array}{l}0,29 \\
0,35\end{array}$ & $\begin{array}{l}0,25 \\
0,21\end{array}$ & $\overline{0,33}$ & 0,10 & $-\overline{0,06}$ & $\begin{array}{c}-0,12 \\
-\end{array}$ & $\begin{array}{l}0,07 \\
0,03\end{array}$ & $\begin{array}{r}0,04 \\
-0,09\end{array}$ & $\begin{array}{r}0,22 \\
-0,02\end{array}$ & $\begin{array}{l}0,17 \\
0,02\end{array}$ \\
\hline HT & $\begin{array}{r}0,04 \\
-0,34\end{array}$ & $\begin{array}{l}-0,16 \\
-0,11\end{array}$ & $\begin{array}{l}0,71 \\
0,68\end{array}$ & $\begin{array}{l}0,81 \\
0,78\end{array}$ & & & $\begin{array}{l}0,39 \\
0,50\end{array}$ & $\begin{array}{l}0,32 \\
0,39\end{array}$ & $\overline{0,19}$ & 0,27 & $\overline{0,12}$ & 0,04 & $\begin{array}{r}0,02 \\
-0,10\end{array}$ & $\begin{array}{l}-0,10 \\
-0,08\end{array}$ & $\begin{array}{r}0,27 \\
-0,10\end{array}$ & $\begin{array}{l}0,07 \\
0,10\end{array}$ \\
\hline LONG & $\begin{array}{r}0,56 \\
-0,01\end{array}$ & $\begin{array}{r}-0,19 \\
0,37\end{array}$ & $\begin{array}{l}0,20 \\
0,15\end{array}$ & $\begin{array}{l}0,26 \\
0,15\end{array}$ & $\begin{array}{l}0,35 \\
0,20\end{array}$ & $\begin{array}{l}0,39 \\
0,19\end{array}$ & & & $\overline{0,19}$ & 0,02 & $-\overline{0,11}$ & $\stackrel{0,09}{-}$ & $\begin{array}{l}-0,02 \\
-0,06\end{array}$ & $\begin{array}{l}0,06 \\
0,02\end{array}$ & $\begin{array}{r}0,14 \\
-0,06\end{array}$ & $\begin{array}{r}-0,10 \\
0,19\end{array}$ \\
\hline LARG & $\overline{0,02}$ & $\begin{array}{c}-0,01 \\
-\end{array}$ & $\overline{0,39}$ & $\begin{array}{l}0,13 \\
-\end{array}$ & $\overline{0,09}$ & $\begin{array}{c}-0,23 \\
-\end{array}$ & $-\overline{0,24}$ & $\begin{array}{c}-0,04 \\
-\end{array}$ & & & $\overline{0,22}$ & $\begin{array}{c}-0,13 \\
-\end{array}$ & $-0,01$ & $-0,29$ & $\overline{0,10}$ & $\begin{array}{l}0,15 \\
-\end{array}$ \\
\hline FLOR & $-0,65$ & $\begin{array}{c}-0,38 \\
-\end{array}$ & $\overline{0,47}$ & $\begin{array}{l}0,39 \\
-\end{array}$ & $\overline{0,48}$ & $\begin{array}{l}0,54 \\
-\end{array}$ & $\overline{0,33}$ & $\begin{array}{l}0,36 \\
-\end{array}$ & $\overline{0,18}$ & $\begin{array}{c}-0,14 \\
-\end{array}$ & & & $-\overline{0,32}$ & $\begin{array}{c}-0,15 \\
-\end{array}$ & $-\overline{0,16}$ & $\begin{array}{c}-0,14 \\
-\end{array}$ \\
\hline$\%$ MS & $\begin{array}{l}0,43 \\
0,63\end{array}$ & $\begin{array}{l}0,43 \\
0,31\end{array}$ & $\begin{array}{l}-0,15 \\
-0,29\end{array}$ & $\begin{array}{l}-0,44 \\
-0,40\end{array}$ & $\begin{array}{l}-0,28 \\
-0,40\end{array}$ & $\begin{array}{l}-0,40 \\
-0,34\end{array}$ & $\begin{array}{r}0,11 \\
-0,15\end{array}$ & $\begin{array}{r}-0,31 \\
0,09\end{array}$ & $-\overline{0,09}$ & $-0,68$ & $-\overline{0,76}$ & $\begin{array}{c}-0,68 \\
-\end{array}$ & & & $\begin{array}{l}0,43 \\
0,25\end{array}$ & $\begin{array}{l}0,14 \\
0,32\end{array}$ \\
\hline RDT & $\begin{array}{l}0,89 \\
0,40\end{array}$ & $\begin{array}{l}0,15 \\
0,32\end{array}$ & $\begin{array}{l}0,75 \\
0,52\end{array}$ & $\begin{array}{l}0,46 \\
0,54\end{array}$ & $\begin{array}{l}0,79 \\
0,52\end{array}$ & $\begin{array}{l}0,52 \\
0,57\end{array}$ & $\begin{array}{l}0,88 \\
0,34\end{array}$ & $\begin{array}{l}0,42 \\
0,12\end{array}$ & 0,44 & $+0,38$ & $\overline{0,02}$ & 0,54 & $\begin{array}{l}0,38 \\
0,16\end{array}$ & $\begin{array}{r}-0,36 \\
0,05\end{array}$ & & \\
\hline
\end{tabular}


TABLEAU 5

Corrélations génétiques et corrélations résiduelles pour les synthétiques cornées $20^{(1)}$ et $4 \times 14^{(2)}$. Corrélations génétiques sous la diagonale, corrélations résiduelles au-dessus de la diagonale).

Genetic and residual correlations for flint synthetics $20^{\prime \prime}$ and $4 \times 14^{(2)}$. (Genetic correlations are below the diagonal; residual correlations above the diagonal.)

\begin{tabular}{|c|c|c|c|c|c|c|c|c|c|c|c|c|c|c|c|c|}
\hline \multirow[b]{2}{*}{ VIG } & \multicolumn{2}{|c|}{ VIG } & \multicolumn{2}{|c|}{$\mathrm{HE}$} & \multicolumn{2}{|c|}{$\mathrm{HT}$} & \multicolumn{2}{|c|}{ LONG } & \multicolumn{2}{|c|}{ LARG } & \multicolumn{2}{|c|}{ FLOR } & \multicolumn{2}{|c|}{$\% \mathrm{MS}$} & \multicolumn{2}{|c|}{ RDT } \\
\hline & & & $-0,03$ & 0,12 & 0,02 & $-0,04$ & 0,12 & 0,14 & - & 0,25 & - & $-0,36$ & 0,11 & 0,35 & 0,18 & 0,24 \\
\hline $\mathrm{HE}$ & $-0,22^{(1)}$ & $-0,02$ & & & 0,52 & 0,53 & 0,31 & 0,28 & - & 0,48 & - & $-0,05$ & $-0,06$ & 0,15 & 0,13 & 0,12 \\
\hline HT & $-0,17$ & $-0,22$ & 0,75 & 0,94 & & & 0,41 & 0,49 & - & 0,20 & - & 0,11 & $-0,06$ & 0,00 & 0,07 & 0,04 \\
\hline LONG & 0,26 & 0,37 & 0,08 & 0,55 & 0,18 & 0,62 & & & & 0,32 & - & $-0,12$ & $-0,05$ & 0,09 & 0,10 & 0,19 \\
\hline LARG & - & $-0,12$ & - & 0,55 & - & 0,52 & - & 0,19 & & & - & $-0,24$ & - & $-0,24$ & - & 0,22 \\
\hline FLOR & - & $-0,40$ & - & 0,66 & - & 0,68 & - & 0,33 & - & 0,64 & & & - & $-0,46$ & - & $-0,05$ \\
\hline$\% \mathrm{MS}$ & 0,61 & 0,17 & $-0,32$ & $-0,43$ & $-0,28$ & $-0,44$ & $-0,06$ & $-0,20$ & - & $-0,57$ & - & $-0,65$ & - & - & 0,39 & 0,13 \\
\hline RDT & 0,54 & 0,01 & 0,52 & 0,82 & 0,65 & 0,86 & 0,74 & 0,71 & - & 0,58 & - & 0,56 & 0,45 & $-0,56$ & - & - \\
\hline
\end{tabular}

TABLEAU 6

Corrélations génétiques faisant intervenir les caractères chimiques pour les synthétiques $21^{(1)}, 3 \times 13^{(2)}, 4 \times 14^{(3)}, 5 \times 9^{(4)}$. Genetic correlations with chemical characters for synthetics $21^{(1)}, 3 \times 13^{(2)}, 4 \times 14^{(3)}, 5 \times 9^{(4)}$.

\begin{tabular}{|c|c|c|c|c|c|c|c|c|c|c|}
\hline & VIG & $\mathrm{HE}$ & $\mathrm{HT}$ & LONG & LARG & $\% \mathrm{MS}$ & FLOR & RDT & $\mathrm{ADF}$ & PROT \\
\hline $\begin{array}{rr}\text { ADF } & (1) \\
& (2) \\
& (3)\end{array}$ & $\begin{array}{r}0,09 \\
-0,36 \\
-0,01\end{array}$ & $\begin{array}{l}0,37 \\
0,09 \\
0,26\end{array}$ & $\begin{array}{l}0,53 \\
0,39 \\
0,99\end{array}$ & $\begin{array}{r}0,27 \\
-0,06 \\
0,22\end{array}$ & $\begin{array}{r}-0,37 \\
0,06 \\
0,01\end{array}$ & $\begin{array}{r}0,80 \\
-0,29 \\
-0,58\end{array}$ & $\begin{array}{r}-0,56 \\
0,40 \\
0,31\end{array}$ & $\begin{array}{l}0,96 \\
0,90 \\
0,46\end{array}$ & & \\
\hline $\begin{array}{r}\text { PROT (1) } \\
(2) \\
(3) \\
(4)\end{array}$ & $\begin{array}{l}0,35 \\
0,44 \\
0,14 \\
0,05\end{array}$ & $\begin{array}{r}0,01 \\
0,02 \\
-0,32 \\
-0,32\end{array}$ & $\begin{array}{r}0,08 \\
-0,37 \\
-0,36\end{array}$ & $\begin{array}{l}-0,09 \\
-0,02 \\
-0,04 \\
-0,33\end{array}$ & $\begin{array}{r}0,58 \\
0,15 \\
-0,05 \\
-\end{array}$ & $\begin{array}{l}-0,52 \\
-0,52 \\
-0,48\end{array}$ & $\begin{array}{l}0,59 \\
0,80 \\
0,48 \\
0,22\end{array}$ & $\begin{array}{l}-0,58 \\
-0,71 \\
-0,49 \\
-0,53\end{array}$ & $\begin{array}{l}-0,55 \\
-0,53 \\
-0,75 \\
-\end{array}$ & \\
\hline $\begin{array}{r}\text { GLUC (1) } \\
(2) \\
(3)\end{array}$ & $\begin{array}{l}-0,32 \\
-0,57 \\
-0,25\end{array}$ & $\begin{array}{l}0,38 \\
0,09 \\
0,54\end{array}$ & $\frac{0,43}{0,63}$ & $\begin{array}{l}0,12 \\
0,19 \\
0,28\end{array}$ & $\begin{array}{r}-0,03 \\
-0,25 \\
0,44\end{array}$ & $\begin{array}{r}-0,28 \\
0,86 \\
0,72\end{array}$ & $\begin{array}{r}0,87 \\
-0,93 \\
-0,87\end{array}$ & $\begin{array}{r}0,79 \\
-0,12 \\
0,63\end{array}$ & $\begin{array}{l}0,33 \\
0,39 \\
0,29\end{array}$ & $\begin{array}{l}-0,64 \\
-0,86 \\
-0,69\end{array}$ \\
\hline
\end{tabular}

matériel corné, ou encore pour la relation rendementvigueur au départ). Ce type de résultat est bien attendu puisque les synthétiques ont des compositions génétiques différentes. Cependant, il est remarquable de constater que certaines corrélations génétiques sont toujours assez fortes :

- entre caractères morphologiques et rendement : des corrélations positives hauteur de l'épi-hauteur totale, hauteur de l'épi-rendement et hauteur totale-rendement.

- entre caractères de précocité et la composition chimique ( $\mathrm{r}$ représentant un coefficient de corrélation) :

$r$ fortement négative entre p. 100 de MS et teneur en glucides solubles,

r positive (négative) entre teneur en MS (date floraison) et teneur en protéines.

Toutefois, cette relation n'existe pas pour la synthétique $5 \times 9$.

- entre rendement et composition chimique :

$r$ fortement positive entre teneur en ADF et rendement pour les synthétiques 21 et $3 \times 13$; plus faible pour la synthétique $4 \times 14$.

$r$ assez fortement négative pour les 4 synthétiques étudiées entre rendement et teneur en protéines.

- entre caractères de composition chimique :

$r$ négative entre ADF et teneur en protéines,

$r$ négative entre teneur en protéines et teneur en glucides solubies.

Ces corrélations assez fortes et stables d'une population à l'autre pourraient signifier l'existence d'effets pléïotropiques et seraient donc difficiles à rompre par sélection.

\section{Discussion du point de vue des critères de sélection}

En moyenne, les caractères morphologiques (hauteur de la plante, hauteur de l'ćpi, dimensions de la feuille de l'épi) sont très liés au rendement. Cependant, parmi ces caractères, la hauteur de la plante et la hauteur de l'épi semblent avoir une valeur plus stable que les dimensions foliaires. Ces résultats tendent à montrer que, pour avoir le rendement maximum en matière sèche, il faut un développement de toutes les parties de la plante et, en particulier, de la tige. Une telle morphologic correspond à une morphologic de génotypes tardifs à floraison. Cependant, il n'y a pas de relation «stable " entre rendement et précocité, ce qui signific que dans l'intervalle de variation étudié, il est possible de créer des génotypes précoces et productifs.

La vigucur au départ scmble en moyenne liée positivement au rendement, de façon plus variable toutefois que la hautcur totale de la plante. Cette notation de vigueur, pratiquéc environ à l'ćpoque où s'cffectue la différenciation de la panicule ( 7 à 9 feuilles visibles), reflète en grande partic des différences de précocité. Elle peut cependant s'avérer cfficace pour réaliser un compromis dans la sélection conjointe pour le rendement et la tencur en matière sèche.

Les corrélations entre critères de qualité et rendement tendent à montrer une opposition rendement-qualité. Elle est très nette pour l'ADF pour les 2 synthétiques cornćes, mais clle l'est moins pour une synthétique dentée.

La teneur en protéines est liće négativement au rendement. La liaison n'est pas très stricte et pourrait permettre 
d'espérer un progrès génétique sur la tencur en protéines sans perte de rendement s'il était possible d'améliorer sensiblement l'héritabilité du premier caractère en réduisant l'erreur expérimentale.

La teneur en glucides solubles, autre facteur de qualité, est liée positivement au rendement, mais probablement par l'intermédiaire de la précocité (p. 100 matière sc̀che), comme le montre l'examen des corrélations rendementp. 100 MS, d'une part, rendement-p. 100 glucides solubles, d'autre part. Elle est sans doute liée de façon négative assez fortc avec la teneur cn amidon qui reflc̀te la participation du grain au rendement total, mais ce caractère n'a pas été mesuré dans le cadre des essais rapportés ici.

La liaison négative observée entre ADF ct protéines, imputable en partic à la variation de précocité, souligne en outre l'existence possible d'un certain niveau de coordination entre des voics métaboliques qui concernent différents compartiments de la plante (partic «grain», partie «non grain $»)$ : la partie non grain pourrait contribuer de façon assez importante à la variation de teneur en protéines.

\section{Corrélations « résiduelles » (intra-descendances)}

Ces corrélations sont aussi importantes à considérer. Par comparaison aux corrélations génétiques, elles peuvent permettre de formuler des hypothèses sur la nature de liaison génétique. En effet, si les corrélations sont de même sens, il est difficile de conclure, mais il est permis de privilégier l'hypothèse de pléiotropie. Si elles sont de sens opposé, l'hypothèse de liaison due au linkage semble plus probable. Enfin, le gain d'information apporté par un caractère pour l'appréciation de la valeur d'un autre est plus grand lorsque ces 2 corrélations sont opposées (VINCOURT \& GALlaIS, 1981). Ces corrélations (tabl. 4, 5) sont en général assez faibles. Cependant, elles sont assez fortes entre hauteur de l'épi et hauteur totale et entre teneur en $\mathrm{ADF}$ et teneur en protéines, c'est-à-dire dans le même sens que les corrélations génétiques correspondantes. Cela renforce l'hypothèse de pléïotropie précédemment formulée pour expliquer les liaisons génétiques observées. La hauteur totale et la hauteur de l'épi peuvent donc être considérées comme des caractères très proches l'un de l'autre. De même, l'opposition teneur en ADF-teneur en protéines semble donc avoir une base physiologique et sera sans doute assez difficile à rompre par la sélection.

\section{Intérêt de la liaison entre caractères pour la sélection sur un caractère}

\section{Examen des possibilités de sélection sur un ou plusieurs caractères pour une réponse sur un autre caractère}

Pour qu'il soit plus efficace de sélectionner sur un caractère lié (dit secondaire) au caractère sélectionné (dit primaire) plutôt que sur ce caractère sélectionné lui-même, il faut que l'héritabilité $h_{2}$ du caractère secondaire soit assez élevée, plus que celle $\left(\mathrm{h}_{1}\right)$ du caractère primaire, et que leur corrélation génétique $\left(\rho_{12}\right)$ soit assez forte, telles que :

$$
\rho_{12} h_{2}>h_{1} \text {. }
$$

Nous avons considéré la réponse à la sélection pour le rendement et la teneur en ADF et en protéines (caractères primaires) en prenant comme caractères secondaires ceux dont les corrélations génétiques avec les caractères primaires étaient les plus élevées. En fait, la relation précédente n'est que rarement vérifiée. Pour le rendement, la sélection indirecte sur la hautcur de l'épi ou la hautcur totale est plus efficace que la sélection directe pour les synthétiques 19 ct 20, là où l'héritabilité cst très faible. De même, dans les synthétiques $3 \times 13$ et 21 , compte tenu de la faible héritabilité de la teneur en ADF et de la forte liaison ADFrendement, il peut être aussi ou plus efficace de mesurer sculement le rendement pour sélectionner au niveau de l'ADF, ce qui était évident compte tenu de la fortc corrélation génétique observée.

Un autre exemple plus intéressant à considérer du point de vue des critères de sélection pour la qualité est la réponse de la teneur en protéines à la sélection pour la tencur en glucides : une contre-sélection de la teneur en glucides peut apporter un progrès corrélatif aussi ou plus important que la sélection directe.

Il n'apparaît donc pas de règle générale pour la sélection indirecte sur un scul caractère. La mesure de plusieurs caractères secondaires liés au caractère primaire ne peut que fairc gagner en information par rapport à la mesure d'un seul caractère. Ainsi les caractères morphologiques associés au rendement et contribuant à améliorer la précision de la valeur génétique estimée pour ce caractère peuvent ĉtre utilisés comme sculs prédicteurs.

Le tableau 7 donne, pour chaque synthétique, le degré de prédiction $d u$ rendement, $r_{(G, \bar{G})}^{2}$, obtenu à partir des caractères mesurés dans la phase levée-floraison. Ces valcurs sont à comparer aux héritabilités univariables $\left(\mathrm{h}_{\mathrm{u}}^{2}\right)$ du rendement données dans le tableau 8 . Il est remarquable que, sauf pour la synthétique $3 \times 13$, la seule considération des caractères morphologiques et de la vigueur au départ apporte autant ou plus d'information que la mesure du rendement lui-même.

Compte tenu de la liaison négative entre taille des organes et tardiveté de floraison, on peut craindre toutefois qu'unc sélection indirccte pour le rendement à partir des seuls caractères morphologiques (hauteur des plantes, dimensions des feuilles) ne soit accompagnée d'une augmentation de tardiveté. La prise en compte de la vigueur au départ atténue cette tendance en particulier pour les synthétiques étudiées en 1981 (Syn 19, Syn 20, Syn $5 \times 9$ ) (tabl. 7)

La sélection indirecte multicaractères, avec les caractères morphologiques observés, apparaît donc en moyenne assez efficace pour le rendement. Elle pourra être utilisée dans certaines situations, par exemple pour réaliser un premier tri dans les premières étapes de la sélection.

\section{Appréciation de la valeur génétique à l'aide de plusieurs caractères}

Nous n'avons considéré que la réponse à la sélection sur le rendement, le p. 100 de MS, la teneur en ADF et la teneur en protéines, l'ensemble des caractères mesurés sur une synthétique donnée étant pris comme variables prédictrices. Pour faciliter l'expression, nous parlerons de sélection univariable pour la sélection directe sur un seul caractère et de sélection multivariable pour la sélection sur un caractère, mais avec la prise en considération du caractère primaire et de différents caractères secondaires. Par rapport à la sélection univariable, le tableau 8 montre que le progrès génétique attendu par sélection « multivariable » est toujours augmenté, et quelquefois fortement (200 p. 100), pour les 3 caractères à héritabilité faible (rendement, teneur en ADF et protéines) ; par contre, pour la teneur en matière sèche, caractère en moyenne plus héritable, l'information apportée par les autres caractères est assez faible. Le gain d'information est d'autant plus fort que l'héritabilité du caractère considéré est plus faible. 
TABLEAU 7

Prédiction du rendement et réponse à la sélection à partir de caractères mesurés durant la période levée-floraison comparée à la prédiction possible

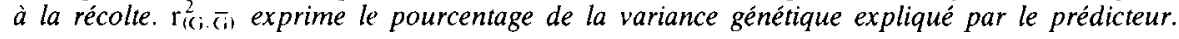

Prediction of dry matter yield and response to selection from characters observed between emergence and flowering as compared to possible prediction at harvest. $\mathrm{r}_{(\mathrm{i}, \overline{(}, \mathrm{i})}^{2}$ represents the percentage of the genetic variance explained by the predictor.

\begin{tabular}{|c|c|c|c|c|}
\hline Synthétiques & Prédicteurs & $r_{i(i, \overline{c i})}^{2}$ & $\Delta \mathrm{G}(\mathrm{t} / \mathrm{ha})$ & $\begin{array}{c}\Delta \mathrm{G}\left(^{*}\right) \text { points } \\
\% \text { MS }\end{array}$ \\
\hline Syn 20 & $\begin{array}{l}\text { A1 } \\
\text { A2 } \\
\text { A3 }\end{array}$ & $\begin{array}{l}0,396 \\
0,579 \\
0,681\end{array}$ & $\begin{array}{l}0,46 \\
0,59 \\
0,60\end{array}$ & $\begin{array}{r}-0,5 \\
0,3 \\
0,1\end{array}$ \\
\hline Syn $4 \times 14$ & $\begin{array}{l}\text { B1 } \\
\text { B2 } \\
\text { B3 }\end{array}$ & $\begin{array}{l}0,502 \\
0,506 \\
0,684\end{array}$ & $\begin{array}{l}1,05 \\
1,05 \\
1,23\end{array}$ & $\begin{array}{l}-1,0 \\
-1,0 \\
-1,2\end{array}$ \\
\hline Syn 19 & $\begin{array}{l}\text { A1 } \\
\text { A2 } \\
\text { A.3 }\end{array}$ & $\begin{array}{l}0,752 \\
0,944 \\
1,0\end{array}$ & $\begin{array}{l}1,00 \\
1,13 \\
1,21\end{array}$ & $\begin{array}{r}-0,6 \\
0,2 \\
-0,1\end{array}$ \\
\hline Syn $3 \times 13$ & $\begin{array}{l}\text { B1 } \\
\text { B2 } \\
\text { B3 }\end{array}$ & $\begin{array}{l}0,213 \\
0,263 \\
0,525\end{array}$ & $\begin{array}{l}0,56 \\
0,62 \\
0,88\end{array}$ & $\begin{array}{r}-0,3 \\
-0,1 \\
0,0\end{array}$ \\
\hline Syn 21 & $\begin{array}{l}\text { B1 } \\
\text { B2 } \\
\text { B3 }\end{array}$ & $\begin{array}{l}0,281 \\
0,323 \\
0,483\end{array}$ & $\begin{array}{l}0,55 \\
0,59 \\
0,73\end{array}$ & $\begin{array}{l}-2,0 \\
-1,7 \\
-1,9\end{array}$ \\
\hline Syn $5 \times 9$ & $\begin{array}{l}\text { A1 } \\
\text { A2 } \\
\text { A3 }\end{array}$ & $\begin{array}{l}0,194 \\
0,306 \\
0,456\end{array}$ & $\begin{array}{l}0,48 \\
0,61 \\
0,74\end{array}$ & $\begin{array}{r}-0,8 \\
-0,3 \\
0,0\end{array}$ \\
\hline
\end{tabular}

(*) Réponse corrélative de la teneur en matière sèche à la sélection pour le rendement Correlated response of dry matter content to selection for yield.
$\mathrm{A} 1=\mathrm{HE}, \mathrm{HP}, \mathrm{LONG}$
B1 = HE, HP, LONG, LARG, FLOR
$\mathrm{A} 2=\mathrm{A} 1, \mathrm{VIG}$
$\mathrm{B} 2=\mathrm{B} 1$, VIG
$\mathrm{A} 3=\mathrm{A} 2, \mathrm{RDT}, \% \mathrm{MS}$
$\mathrm{B} 3=\mathrm{B} 2, \mathrm{RDT}, \% \mathrm{MS}$

TABLEAU 8

Héritabilités $\left(h^{2}\right)$ et réponse à la sélection $(\Delta G)$ en "univariable " $(U)$ et en "multivariable " $(M)$ (La situation multivariable correspond à l'utilisation de toutes les variables comme prédicteurs des valeurs génotypiques d'un caractère).

Heritabilities $\left(h^{2}\right)$ and selection response $(\Delta G)$ in the univariate $(U)$ and multivariate situation $(M)$. (The multivariate situation corresponds to the use of all characters as predictors of the genotypic value for one character.)

\begin{tabular}{|c|c|c|c|c|c|c|c|}
\hline & & Syn 20 & Syn $4 \times 14$ & Syn 19 & Syn $3 \times 13$ & Syn 21 & Syn $5 \times 9$ \\
\hline Rendement & $\begin{array}{l}\mathbf{h}_{\mathrm{U}}^{2} \\
\mathbf{h}_{\mathrm{M}}^{2} \\
\Delta \mathrm{G}_{\mathrm{U}} \\
\Delta \mathrm{G}_{\mathrm{M}}\end{array}$ & $\begin{array}{l}0,12 \\
0,68 \\
0,25 \\
0,61\end{array}$ & $\begin{array}{l}0,54 \\
0,72 \\
1,09 \\
1,25\end{array}$ & $\begin{array}{l}0,23 \\
1 \\
0,55 \\
1,21\end{array}$ & $\begin{array}{l}0,42 \\
0,70 \\
0,79 \\
1,02\end{array}$ & $\begin{array}{l}0,27 \\
0,74 \\
0,54 \\
0,91\end{array}$ & $\begin{array}{l}0,32 \\
0,48 \\
0,62 \\
0,76\end{array}$ \\
\hline$\% \mathrm{MS}$ & $\begin{array}{l}\mathbf{h}_{\mathrm{U}}^{2} \\
\mathbf{h}_{\mathrm{M}}^{2} \\
\Delta \mathrm{G}_{\mathrm{U}} \\
\Delta \mathrm{G}_{\mathrm{M}}\end{array}$ & $\begin{array}{l}0,62 \\
0,67 \\
2,1 \\
2,2\end{array}$ & $\begin{array}{l}0,38 \\
0,61 \\
1,4 \\
1,8\end{array}$ & $\begin{array}{l}0,38 \\
0,60 \\
1,3 \\
1,6\end{array}$ & $\begin{array}{l}0,65 \\
0,72 \\
2,3 \\
2,5\end{array}$ & $\begin{array}{l}0,64 \\
0,76 \\
3,0 \\
3,3\end{array}$ & $\begin{array}{l}0,55 \\
0,57 \\
2,0 \\
2,0\end{array}$ \\
\hline $\mathrm{ADF}$ & $\begin{array}{l}\mathrm{h}_{\mathrm{U}}^{2} \\
\mathrm{~h}_{\mathrm{M}}^{\mathrm{M}} \\
\Delta \mathrm{G}_{\mathrm{U}} \\
\Delta \mathrm{G}_{\mathrm{M}}\end{array}$ & $\begin{array}{l}- \\
-\end{array}$ & $\begin{array}{l}0,34 \\
0,53 \\
0,67 \\
0,84\end{array}$ & $\begin{array}{l}- \\
-\end{array}$ & $\begin{array}{l}0,20 \\
0,89 \\
0,56 \\
0,81\end{array}$ & $\begin{array}{l}0,10 \\
0,77 \\
0,42 \\
0,76\end{array}$ & $\begin{array}{l}- \\
- \\
-\end{array}$ \\
\hline PROT & $\begin{array}{l}\mathrm{h}_{\mathrm{U}}^{2} \\
\mathrm{~h}_{\mathrm{M}}^{2} \\
\Delta \mathrm{G}_{\mathrm{U}} \\
\Delta \mathrm{G}_{\mathrm{M}}\end{array}$ & $\frac{-}{-}$ & $\begin{array}{l}0,41 \\
0,55 \\
0,35 \\
0,40\end{array}$ & $\begin{array}{l}- \\
-\end{array}$ & $\begin{array}{l}0,37 \\
0,96 \\
0,27 \\
0,44\end{array}$ & $\begin{array}{l}0,31 \\
0,77 \\
0,16 \\
0,25\end{array}$ & $\begin{array}{l}0,14 \\
0,31 \\
0,17 \\
0,25\end{array}$ \\
\hline
\end{tabular}


Ce résultat illustre l'intérêt de la prise en considération de plusieurs caractères pour la sélection sur un seul caractère. Pour les synthétiques 19 et 20 , la sélection sur le rendement seul était assez inefficace (par suite d'une plus grande erreur expérimentale), mais la considération des caractères de morphologie, liés génétiquement au rendement et plus héritable que le rendement lui-même, permet une sélection plus efficace. La mesure de tels caractères est donc une sorte de sécurité.

Le gain d'information par la sélection « multivariable », par rapport à la sélection « univariable » peut encore s'illustrer par la comparaison des héritabilités correspondant à ces 2 situations (tabl. 8). L'héritabilité multivariable est toujours très augmentée et peut atteindre des valeurs très élevées, même pour le rendement. Cette augmentation d'héritabilité peut s'exprimer par le nombre de répétitions qu'il aurait fallu réaliser pour avoir le même progrès par la mesure d'un seul caractère. Nous ne considérerons ce problème que pour le rendement. Pour les synthétiques 19, 20 et 21 à héritabilités faibles, il aurait fallu plus de 15 répétitions pour passer à une héritabilité de l'ordre de 0,70 ! Le gain en répétitions est évidemment plus faible lorsque l'héritabilité de départ est plus élevée. Ainsi pour les synthétiques $3 \times 13,4 \times 14$ et $5 \times 9$, le gain n'est que de 1 à 4 répétitions, mais il y a toujours eu gain, même en ne mesurant que les caractères morphologiques.

La mesure de caractères morphologiques ou chimiques associés au rendement peut donc apporter l'équivalent de répétitions supplémentaires. Le problème est de savoir ce qui sera le moins coûteux ou le plus facilement réalisable : mesurer 1 ou 2 caractères de plus ou réaliser 3-4 répétitions supplémentaires? En fait, cette équivalence n'est pas toujours possible et l'exemple des synthétiques 19 et 20 montre que l'accroissement du nombre de répétitions, dans des limites « raisonnables", ne pourra pas toujours compenser l'information, la sécurité, susceptible d'être apportée par certains caractères associés (par exemple, la hauteur de la plante). Ainsi, une attaque de pyrale ou une verse importante après floraison peut compromettre des mesures de rendement; si les hauteurs de plantes ont été mesurées à la floraison, une sélection pour le rendement sera quand même possible.

Dans la mesure où la sélection est multicaractères, c'està-dire si elle porte simultanément sur plusieurs caractères, alors tous les caractères «primaires » peuvent déjà être utilisés pour prévoir la valeur génétique de chaque caractère. L'efficacité de cette prédiction dépendra des liaisons génétiques et environnementales entre les caractères. Ainsi, pour toutes les synthétiques étudiées, il existe des relations entre le rendement et la qualité, l'un peut donc être utilisé pour « affiner » la prédiction de l'autre. Mais si les caractères sont liés négativement, tout progrès sur l'un tendra à s'opposer à un progrès sur l'autre et le progrès en valeur économique pourra être faible (cas du rendement et de la teneur en ADF pour les synthétiques 21 et $3 \times 13$ ).

Un inconvénient de l'utilisation comme prédicteurs de caractères primaires en liaison négative est que la méthode de calcul utilisée peut pénaliser les unités candidates à la sélection présentant sur le plan phénotypique une combinaison intéressante de ces caractères. La prise en compte de la liaison génétique négative tend à rapprocher de l'axe de cette liaison les observations qui s'en écartent au niveau phénotypique et qui intéressent fortement le sélectionneur. Il faudrait donc développer une autre méthode de calcul permettant de détecter et de favoriser les associations horstype.

\section{Réponses corrélatives}

Nous avons considéré les réponses corrélatives du rendement, de la teneur en matière sèche, de la teneur en ADF et de la teneur en protéines par sélection sur chacun de ces caractères, en utilisant l'information apportée par toutes les variables mesurées (tabl. 9). Le comportement des 4 synthétiques étudiées est très proche.

- Une sélection sur le rendement seul se traduira en moyenne par une augmentation de tardiveté, une augmentation de la teneur en ADF et une diminution de la teneur en protéines.

- Une sélection pour la précocité fait plus ou moins perdre en rendement, mais elle permet une diminution de la teneur en $\mathrm{ADF}$ et une augmentation de la teneur en protéines, ce qui confirme une observation déjà réalisée par GALLAIS et al. (1976), à savoir que les génotypes plus tardifs sont plus riches en fibres et que les plus précoces sont plus riches en protéines.

- Une sélection contre l'ADF pour améliorer la digestibilité se traduira par une assez forte diminution du rendement ; il apparaît donc difficile de concilier digestibilité et rendement par l'utilisation de l'ADF. GALLAIS et al. (1976) avaient déjà montré une tendance à une telle liaison et concluaient que la digestibilité in vitro, moins liée négativement au rendement, était un meilleur critère de valeur alimentaire que la teneur en fibres ou en cellulose.

- La sélection pour la teneur en protéines conduira à une certaine diminution du rendement, avec diminution de la teneur en $\mathrm{ADF}$, mais un certain compromis teneur en protéines-rendement semble possible.

\section{TABLEAU 9}

Réponses corrélatives attendues par sélection sur un caractère en utilisant l'information apportée par toutes les variables mesurées. Expected correlated responses to selection on one character by the use of all characters as predictor of the genotypic value.

\begin{tabular}{|c|c|c|c|c|c|}
\hline $\begin{array}{l}\text { Sélection } \\
\text { sur }\end{array}$ & $\begin{array}{l}\text { Réponse } \\
\text { sur }\end{array}$ & RDT & $\%$ MS & $\mathrm{ADF}$ & PROT \\
\hline RDT & $\begin{array}{l}(1) \\
(2) \\
(3) \\
(4)\end{array}$ & $\begin{array}{l}1,2 \\
1,0 \\
0,9 \\
0,8\end{array}$ & $\begin{array}{r}-1,3 \\
0,0 \\
-1,7 \\
-0,2\end{array}$ & $\begin{array}{l}0,46 \\
0,24 \\
0,61 \\
-\end{array}$ & $\begin{array}{l}-0,24 \\
-0,12 \\
-0,11 \\
-0,15\end{array}$ \\
\hline$\%$ MS & & $\begin{array}{r}-0,9 \\
0,0 \\
-0,5 \\
-0,1\end{array}$ & $\begin{array}{l}1,7 \\
2,4 \\
3,3 \\
2,0\end{array}$ & $\begin{array}{c}-0,34 \\
-0,31 \\
-0,50 \\
-\end{array}$ & $\begin{array}{l}0,24 \\
0,31 \\
0,18 \\
0,10\end{array}$ \\
\hline $\operatorname{ADF}(*)$ & & $\begin{array}{c}-0,70 \\
-0,74 \\
-0,76 \\
-\end{array}$ & $\begin{array}{l}0,70 \\
1,0 \\
2,2 \\
-\end{array}$ & $\begin{array}{c}-0,84 \\
-0,80 \\
-0,76 \\
-\end{array}$ & $\begin{array}{l}0,31 \\
0,34 \\
0,14 \\
-\end{array}$ \\
\hline PROT & & $\begin{array}{l}-0,70 \\
-0,30 \\
-0,40 \\
-0,46\end{array}$ & $\begin{array}{l}1,0 \\
1,7 \\
2,4 \\
0,8\end{array}$ & $\begin{array}{c}-0,64 \\
-0,61 \\
-0,41 \\
-\end{array}$ & $\begin{array}{l}0,40 \\
0,44 \\
0,25 \\
0,24\end{array}$ \\
\hline
\end{tabular}

(*) Coefficient négatif.

(1) $=$ Syn $4 \times 14,(2)=\operatorname{Syn} 3 \times 13,(3)=\operatorname{Syn} 21$,

(4) $=\operatorname{Syn} 5 \times 9$. 


\section{CONCLUSIONS}

Du point de vue des critères de sélection du maïs fourrage, cette étude sur plusieurs populations synthétiques précise assez nettement « l'idéotype » pour la production de matière sèche. Elle confirme le fait que pour avoir le rendement maximum en matière sèche, il faut un développement important de la tige. Elle montre qu'il est possible pour une précocité d'ensilage donnée, d'améliorer le rendement en matière sèche de façon plus efficace en associant au rendement certains caractères morphologiques tels que hauteur de l'épi ou hauteur de la plante. Elle confirme aussi une tendance à une opposition entre rendement et qualité appréciée par la teneur en ADF et la teneur en protéines. Pour la valeur alimentaire (digestibilité, quantité consommée), il faudrait donc trouver d'autres critères, moins liés négativement au rendement. C'est peut-être le cas de la digestibilité in vitro (GALLAIS et al., 1976), ou de la digestibilité de la cellulose. De plus, diminuer la teneur en ADF présente aussi l'inconvénient d'augmenter la sensibilité à la verse à maturité, voire même en végétation.

L'absence de verse en végétation ou de verse à la récolte (ou l'absence de notations discriminantes) sur l'ensemble des essais étudiés ne permet pas de préciser l'importance des liaisons entre ce caractère et les différents caractères considérés dans cette étude. On peut toutefois craindre une liaison positive entre hauteur (surtout hauteur de l'épi) et sensibilité à la verse et une liaison négative entre la teneur en $\mathrm{ADF}$ et la résistance à la verse. La recherche justifiée de la résistance à la verse à la récolte risque donc de limiter les possibilités de progrès tant au niveau du rendement qu'au niveau de la valeur alimentaire.

Du point de vue méthodologique, cette étude montre l'efficacité de caractères liés à un autre caractère pour apprécier la valeur génétique de celui-ci. Des caractères comme la hauteur de la plante ou de l'épi semblent pouvoir apporter plus d'information que l'augmentation du nombre de répétitions, dans la mesure où ils sont plus héritables que le rendement lui-même et en assez forte liaison génétique avec lui. De plus, ils apportent un aspect de sécurité qui ne peut pas être compensé par l'augmentation du nombre de répétitions. La prise en considération des corrélations génétiques et environnementales entre caractères dans la sélection du maîs fourrage apparaît donc utile et efficace, particulièrement pour la pondération biométrique des caractères liés à la production de matière sèche.

Reçu le 2 juillet 1982. Accepté le 11 avril 1983.

\section{RÉFÉRENCES BIBLIOGRAPHIQUES}

Gallais A., 1973. Sélection pour plusieurs caractères. Synthèse critique et généralisation. Ann. Amélior. Plantes, 23, 183-208.

Gallais A., Pollacsek M., Huguet L., 1976. Possibilités de sćlection du maïs en tant que plante fourragère. Ann. Amélior. Plantes, 26, 591-605.

Gallais A., Vincourt P., Huguet L., 1981. Objectifs et critères de sélection du maïs fourrage. $X^{\ell}$ Congrès de la Section Eucarpia Maïs
Sorgho, Montreux, sept. 81.

Rouvier R., 1969. Contribution à l'étude des index de sélection sur plusieurs caractères. Thèse Doctorat $3^{\mathrm{c}}$ cycle. Fac. Sci. Paris.

Vincourt P., Gallais A., 1981. Application of selection index in maize silage breeding Proc. 4th Meeting of the Section Eucarpia "Biometrics in Plant Breeding". ed. A. Gallais, Lusignan, 285294.

\section{ANNEXE}

- Estimation des matrices de variances-covariances entre familles et résiduelles.

La table simplifiée de l'analyse multivariable de la variance est la suivante, b étant le nombre de répétitions, $\Sigma_{\mathrm{gg}}$ la matrice des variances-covariances entre familles et $\Sigma_{\mathrm{ce}}$ la matrice de variances-covariances résiduelles:

\begin{tabular}{lll}
\hline $\begin{array}{c}\text { Source } \\
\text { de variation }\end{array}$ & $\mathrm{PM}$ & $\begin{array}{c}\text { Espérance de la matrice } \\
\text { des produits moyens } \\
\text { des écarts (PM) }\end{array}$ \\
\hline $\begin{array}{l}\text { Famille } \\
\text { Résiduelle }\end{array}$ & $\mathrm{PM}_{\mathrm{g}}$ & $\mathrm{\Sigma M}_{\mathrm{cec}}+\mathrm{b} \Sigma_{\mathrm{gg}}$ \\
\hline
\end{tabular}

$\Sigma_{g g}=\left(\mathrm{PM}_{\mathrm{g}}-\mathrm{P} \mathrm{M}_{\mathrm{v}}\right) / \mathrm{b}$
En l'absence d'interaction génotype $\times$ milieu : cette estimation de $\Sigma_{\mathrm{gg}}$ est une estimation de $\boldsymbol{\Sigma}_{\mathrm{gp}}$ (matrice des covariances entre les valeurs phénotypiques et les valeurs phénotypiques).

Pour décrire la variation totale entre parcelles:

$$
\Sigma_{\mathrm{pp}}=\Sigma_{\mathrm{gg}}+\Sigma_{\mathrm{cc}}
$$

et pour le calcul du progrès génétique par sélection entre familles :

$$
\Sigma_{\mathrm{pp}}=\Sigma_{\mathrm{gg}}+\left(\Sigma_{\mathrm{cc}}\right) / \mathrm{b} \text {. }
$$

